\title{
Benefits Beyond Headache Days With OnabotulinumtoxinA Treatment: A Pooled PREEMPT Analysis
}

\author{
Hans-Christoph Diener - David W. Dodick - Richard B. Lipton · \\ Aubrey Manack Adams · Ronald E. DeGryse - Stephen D. Silberstein
}

Received: July 27, 2020 / Accepted: September 14, 2020 / Published online: October 7, 2020

(C) The Author(s) 2020

\section{ABSTRACT}

Introduction: The double-blind, phase 3 PREEMPT trials demonstrated the efficacy and tolerability of onabotulinumtoxinA for headache prevention in adults with chronic migraine. This post hoc analysis evaluated the effect of onabotulinumtoxinA on clinically meaningful changes in headache severity, headache-related impact, and quality of life.

Methods: Pooled, 24-week data were used to determine percentages of patients meeting responder criteria for the change in headache days $(\geq 50 \%$ reduction in headache-day frequency), Headache Impact Test (HIT-6; $\geq$

H.-C. Diener $(\bowtie)$

Institute for Medical Informatics, Biometry and Epidemiology, University of Duisburg-Essen, Essen, Germany

e-mail: h.diener@uni-essen.de

D. W. Dodick

Mayo Clinic, Phoenix, AZ, USA

R. B. Lipton

Albert Einstein College of Medicine, Bronx, NY, USA

A. Manack Adams

Allergan, an AbbVie Company, Irvine, CA, USA

R. E. DeGryse

AbbVie, Irvine, CA, USA

S. D. Silberstein

Thomas Jefferson University, Philadelphia, PA, USA 5-point improvement), MSQ Role FunctionRestrictive (MSQ-RFR; $\geq 10.9$-point improvement), and Average Daily Headache Severity (ADHS; $\geq 1$-point improvement on a 4-point ordinal scale $[0=$ no pain, $3=$ severe pain $]$ ).

Results: In the pooled analysis population $(N=1384$; onabotulinumtoxinA, $n=688$; placebo, $n=696)$, significantly more patients treated with onabotulinumtoxinA compared with placebo were responders on HIT-6 (40.8 vs. $25.3 \%$ ), MSQ-RFR (59.0 vs. $40.2 \%$ ), and ADHS (35.5 vs. $22.4 \%$ ) measures, and achieved traditional $\geq 50 \%$ reduction in headache days ( 44.8 vs. $34.2 \%$; all $P<0.001$ ). At least one responder criterion was met by $72.1 \%$ and $56.6 \%$ of onabotulinumtoxinA- and placebo-treated patients, respectively; all four were met by $20.4 \%$ and $8.6 \%$, respectively $(P<0.001)$. Linear regression analysis showed that approximately $20 \%$ of the variance in HIT- 6 and MSQRFR improvement was explained by improvement in headache days.

Conclusions: Treatment with onabotulinumtoxinA for 24 weeks was associated with clinically meaningful benefits beyond reduction in headache days; including reductions in headache severity and headache-related impact, and improved quality of life. While $45 \%$ of patients met responder criteria for monthly headache days, over $70 \%$ had clinically meaningful improvements on at least one outcome measure. 
Trial Registration: ClinicalTrials.gov identifier, NCT00156910 (PREEMPT 1) and NCT00168428 (PREEMPT 2)

Keywords: Botulinum toxin type A; Chronic migraine; Quality of life; Responder rate

\section{Key Summary Points}

Why carry out this study?

The proportion of patients who achieve at least a $50 \%$ reduction in headache days is said to be $50 \%$ headache responders, and, at times, this criterion is used as an empirical review for continuing or discontinuing preventive therapy.

However, it is recognized that $50 \%$ responder rates may not fully capture the benefits of preventive treatment.

Therefore, we conducted a post hoc analysis of data from the PREEMPT trials to evaluate the effect of onabotulinumtoxinA on clinically meaningful changes in headache severity, headache-related impact, and quality of life.

\section{What was learned from the study?}

Measurement of the change in the number of headache days did not fully capture the treatment benefit associated with 24 weeks of onabotulinumtoxinA treatment for chronic migraine.

When taking into account clinically meaningful improvements of impact, function, and severity, we observed a more accurate reflection of the comprehensive benefit of onabotulinumtoxinA.

\section{DIGITAL FEATURES}

This article is published with digital features to facilitate understanding of the article. To view digital features for this article go to https://doi. org/10.6084/m9.figshare.12951572.

\section{INTRODUCTION}

Patients with chronic migraine (CM) experience disabling migraine attacks that significantly affect their quality of life and interfere with their ability to perform daily activities $[1,2]$. $\mathrm{CM}$, defined as at least 15 headache days/ month, eight of which fulfill migraine criteria [3], is a neurological disease distinct from episodic migraine (EM), which is characterized by fewer than 15 headache days/month. Headache phenotype changes with transition from EM to $\mathrm{CM}$. This explains why not all headache days in $\mathrm{CM}$ fulfill migraine criteria. CM is also associated with a greater frequency and severity of migraine-associated disability [4]. In addition to enduring more headache days each month, patients with $\mathrm{CM}$ also experience a greater prevalence of comorbidities (e.g., chronic pain disorders, anxiety, depression) compared with those with EM $[1,5]$.

The randomized Phase 3 REsearch Evaluating Migraine Prophylaxis Therapy (PREEMPT 1 and PREEMPT 2) pivotal trials were identically designed to evaluate the clinical efficacy and safety of onabotulinumtoxinA for preventive treatment in adult patients 18 to 65 years of age with CM [6, 7]. Results of these trials established the clinical efficacy, tolerability, and safety of onabotulinumtoxinA for CM, as treatment with onabotulinumtoxinA resulted in significant improvements in a variety of efficacy endpoints, including change from baseline in the frequency of headache days throughout the 24-week double-blind treatment period. In addition, the proportion of patients who achieved at least a 50\% reduction from baseline in headache days, the traditional response threshold used in clinical trials of preventive treatments for $\mathrm{CM}[8,9]$, was significantly greater with onabotulinumtoxinA vs. placebo [10].

The proportion of patients who achieve at least a $50 \%$ reduction in headache days is said to be $50 \%$ headache responders, and, at times, this criterion is used as an empirical review for continuing or discontinuing preventive therapy. However, it is recognized that $50 \%$ responder rates may not fully capture the 
benefits of preventive treatment [11]. For example, a patient may improve from a disabling 20 severe headache days per month to 11 moderate headache days per month. Despite this considerable clinical benefit, such a patient would not be considered a responder because headache days were not reduced by $50 \%$, and might lose access to beneficial treatment [12].

Recognizing these considerations, the American Headache Society recently published a position paper on the management of migraine that provides a series of alternative ways of identifying patients who are benefiting from ongoing treatments, including assessments of disability, disease impact, and healthrelated quality of life [13]. In the spirit of those recommendations, we conducted a post hoc analysis of data from the PREEMPT trials that assessed a series of alternative measures of treatment response. In addition to the 50\% headache day responder definition, we also evaluated the proportions of patients who met responder criteria for three additional validated outcome measures: 6-item Headache Impact Test (HIT-6) total scores, Migraine-Specific Quality of Life Questionnaire (MSQ) Role Function-Restrictive (RFR) domain scores, and Average Daily Headache Severity (ADHS) score.

\section{METHODS}

\section{Study Design}

Methods for the PREEMPT 1 and 2 trials have been reported previously $[6,7]$. In this post hoc analysis, pooled, 24 -week data $[6,7]$ were analyzed. PREEMPT 1 was conducted at 56 North American sites from January 2006 to July 2008, and PREEMPT 2 was conducted at 50 North American and 16 European sites from February 2006 to August 2008. Both trials consisted of a 28-day baseline screening period, followed by a 24-week randomized, double-blind, placebocontrolled phase (two treatment cycles) and a 32-week open-label period during which all patients received onabotulinumtoxinA. During double-blind treatment, patients were randomized to receive intramuscular injections of onabotulinumtoxinA or placebo on day 0 and at week 12, and were administered open-label onabotulinumtoxinA injections at weeks 24,36 , and 48.

Both trials discussed in this article were conducted in accordance with the Declaration of Helsinki Code of Federal Regulations and Good Clinical Practices and US requirements of public registration (NCT00156910 [PREEMPT 1] and NCT00168428 [PREEMPT 2]). Investigators obtained approval from the independent ethics committee or local institutional review board at each investigational site prior to study initiation. Written informed consent was obtained from each randomized patient.

\section{Participants}

Men and women were eligible for study participation if they were 18 to 65 years of age with a history of migraine meeting the diagnostic criteria listed in the 2004 International Classification of Headache Disorders, second edition (ICHD-2), Section 1, Migraine [14], except for "complicated migraine [10]." During the 28-day baseline screening period, patients were required to provide diary data on at least 20 days and to have had at least 15 headache days (defined as a calendar day consisting of $4 \mathrm{~h}$ of continuous headache), of which at least 50\% were migraine or probable migraine days (referred to hereafter as migraine days), and to have had four or more distinct headache episodes each lasting at least $4 \mathrm{~h}$. Patients were excluded if they had used any headache prophylactic medication within 4 weeks prior to the study start at baseline, had previous exposure to any botulinum toxin serotype, or had a positive urine pregnancy test [10].

\section{Efficacy Assessments}

Patients were evaluated based on the following outcome measures: (1) headache days, defined as a calendar day on which the patient reported at least four continuous hours of headache in the headache diary; (2) headache impact, measured with the HIT-6 total score [15]; (3) healthrelated quality of life, measured with the MSQRFR score [16, 17]; and (4) ADHS, based on 
patient-rated headache severity on a 4-point ordinal scale $(0=$ no pain to $3=$ severe pain), with the ADHS score defined as $3^{*}$ severe headache days $+2^{*}$ moderate headache days $+1^{*}$ mild headache day/28. While improvement from baseline involved decreased scores for HIT-6, it involved increased scores for MSQ.

Treatment responders at 24 weeks were determined by four criteria, which align with established values for clinically meaningful changes or minimally important differences reported in the literature. The first is headache days: the proportion of patients achieving at least a 50\% reduction in headache day frequency [18]. Second is headache impact: a within-group reduction in HIT-6 score of at least 5 points from baseline, which has previously been defined as clinically meaningful $[19,20]$. The third is function: the proportion of patients achieving clinically meaningful improvement of at least 10.9 points from baseline in the MSQ-RFR domain score; this magnitude of change was previously established as a within-group minimally important difference $[21,22]$ and also lies above the clinically meaningful threshold for CM (6-point change at month 3) established in a recent psychometric validation study [23]. The MSQ is a psychometrically valid tool to reliably measure the impact of migraine, and the RFR domain measures how performance of daily activities is limited by migraines [17]. In the current analysis, we chose to focus on the MSQ-RFR domain to assess participants' functional limitations attributable to migraine. The fourth criterion is headache severity: in line with a previous pooled analysis of PREEMPT data, a responder with respect to headache severity (ADHS) was defined as a patient who achieved at least a 1-point improvement in ADHS score across the assessment period (e.g., a reduction in headache severity from severe to moderate) [11].

\section{Safety Assessments}

Adverse events (AEs) were reported for the pooled PREEMPT population.

\section{Statistical Analyses}

Fisher's exact test was used to evaluate differences between treatment groups. Missing values for all outcome measures were estimated using a modified last-observation-carried-forward (mLOCF) approach. Use of this method for imputation is consistent with previous analyses of PREEMPT data (vs. other methods, such as multiple imputation or mixed-model repeatmeasures), and has the advantage of allowing the use of the same imputed score for a patient across multiple analyses, even though it is used herein only for comparison of proportions. Proportions of patients meeting responder status in at least one, at least two, at least three, and all four categories were calculated and summarized descriptively. Linear regression analysis was conducted to evaluate the relationship between change in headache days at week 24 and (1) change in HIT-6 score at week 24 and (2) change in MSQ-RFR score at week 24. Venn diagrams were constructed for the proportion of patients meeting responder status for the four outcome measures included in the analysis. Data are summarized separately for the onabotulinumtoxinA and placebo groups.

The analyses reported herein are exploratory, post hoc analyses, without prespecified multiplicity adjustments. However, a conservative Bonferroni adjustment was used when considering the significance of treatment differences for the eight proportions for which $P$ values were calculated. Specifically, the critical level could be lowered from 0.05 to $0.006(0.05 / 8=$ $0.00625)$.

\section{RESULTS}

\section{Patients and Baseline Characteristics}

The pooled analysis population included 1384 patients randomized to onabotulinumtoxinA $(n=688)$ or placebo $(n=696)$. Baseline patient demographics and characteristics were similar between treatment groups and are shown in Table 1 [24]. 
Table 1 Pooled PREEMPT baseline patient demographics and characteristics

\begin{tabular}{|c|c|c|}
\hline & $\begin{array}{l}\text { OnabotulinumtoxinA } \\
(n=688)\end{array}$ & $\begin{array}{l}\text { Placebo } \\
(n=696)\end{array}$ \\
\hline $\begin{array}{l}\text { Age, mean }(\mathrm{SD}), \\
\text { years }\end{array}$ & $41.1(10.4)$ & $41.5(10.7)$ \\
\hline Female, \% & 87.6 & 85.2 \\
\hline White, \% & 89.7 & 90.5 \\
\hline $\begin{array}{l}\text { Frequency of } \\
\text { headache days, } \\
\text { mean }(\mathrm{SD})\end{array}$ & $19.9(3.7)$ & $19.8(3.7)$ \\
\hline $\begin{array}{l}\text { Frequency of } \\
\text { moderate/severe } \\
\text { headache days, } \\
\text { mean (SD) }\end{array}$ & $18.1(4.1)$ & $18.0(4.3)$ \\
\hline $\begin{array}{l}\text { HIT-6 total score, } \\
\text { mean }(\mathrm{SD})\end{array}$ & $65.5(4.1)$ & $65.4(4.3)$ \\
\hline \multicolumn{3}{|c|}{ MSQ domain score, mean (SD) } \\
\hline $\begin{array}{l}\text { Role Function- } \\
\text { Restrictive }\end{array}$ & $38.5(16.6)$ & $38.7(17.3)$ \\
\hline $\begin{array}{l}\text { Role Function- } \\
\text { Preventive }\end{array}$ & $56.0(21.2)$ & $56.1(21.7)$ \\
\hline $\begin{array}{l}\text { Emotional } \\
\text { Function }\end{array}$ & $42.1(24.1)$ & $42.4(25.0)$ \\
\hline
\end{tabular}

HIT-6 6-item Headache Impact Test, MSQ MigraineSpecific Quality of Life Questionnaire, PREEMPT Phase 3 REsearch Evaluating Migraine Prophylaxis Therapy, $S D$ standard deviation

\section{Responder Analysis}

Compared with placebo, significantly more patients treated with onabotulinumtoxinA were responders with regard to change in the number of headache days, HIT-6 total scores, MSQ-RFR domain scores, and ADHS scores at 24 weeks $(P<0.001$, onabotulinumtoxinA vs. placebo for each of the four criteria; Fig. 1). A summary of the headache day responder status $(\geq 50 \%$ reduction in headache days at week 24) comparing mLOCF and observed data analysis is available in a companion article in this journal (Silberstein et al., 2020; Pain and Therapy).

The majority $(72.1 \%)$ of onabotulinumtoxinA-treated patients met at least one of the response criteria, compared with only $44.8 \%$ of onabotulinumtoxinA-treated patients who were considered responders when the traditional threshold of $\mathrm{a} \geq 50 \%$ reduction from baseline in monthly headache days was applied. The responder definition that showed the highest response rate was achievement of $\mathrm{a} \geq 10.9$ point improvement from baseline in the MSQRFR domain score (59\%). Figure 2 shows the proportions of patients who met responder status for at least one, two, and three responder criteria and those who met all four responder criteria. A significantly greater proportion of onabotulinumtoxinA-treated patients compared with placebo-treated patients met at least one to all four responder criteria $(P<0.001$ for onabotulinumtoxinA vs. placebo, all comparisons). More than one in three patients treated with onabotulinumtoxinA qualified as responders on three or more outcomes, and one in five qualified as responders on all outcomes (Fig. 2).

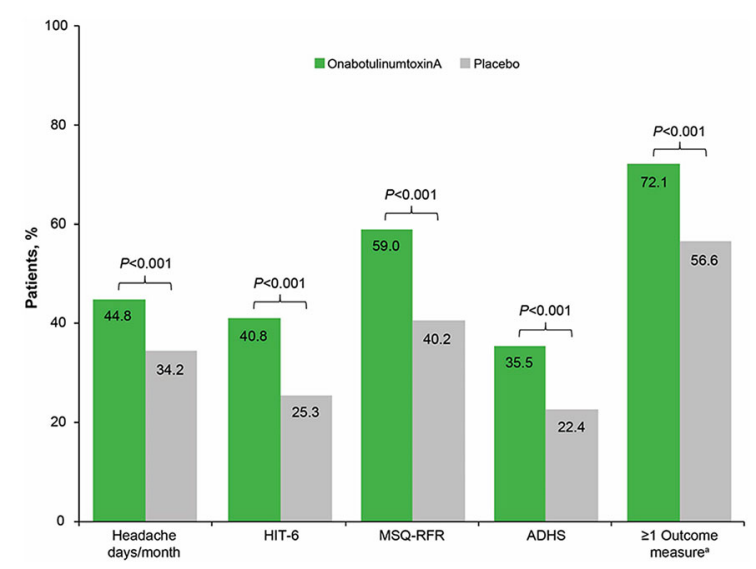

Fig. 1 Responder rates for various outcome measures (mLOCF) for weeks $21-24$ relative to the pretreatment baseline. ${ }^{a}$ Includes any patient who achieved at least one of these four criteria: at least a $50 \%$ reduction in headache days, clinically meaningful change in the HIT-6 total score, MSQ-RFR domain score, or headache severity at week 24. ADHS Average Daily Headache Severity, HIT-6 6-item Headache Impact Test, MSQ-RFR Migraine-Specific Quality of Life Questionnaire Role Function-Restrictive 


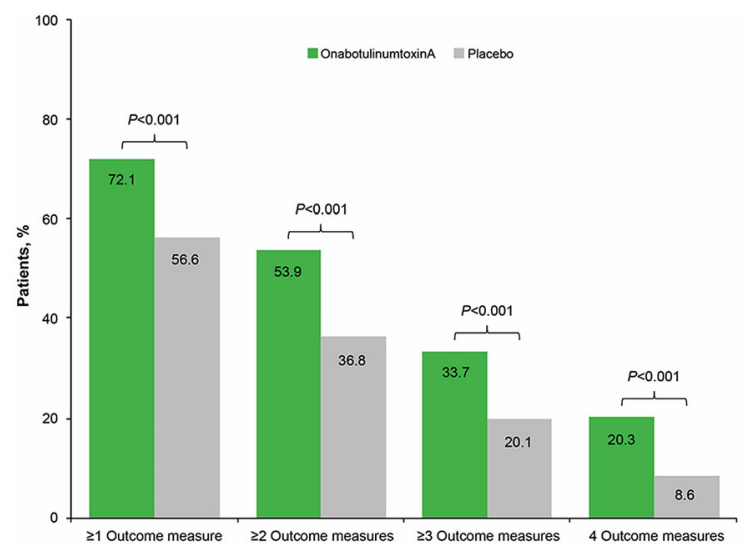

Fig. 2 Percentage of patients who were responders on $\geq 1, \geq 2$, $\geq 3$, and 4 outcome measures. Any patient who achieved at least one of these four outcome measures $-50 \%$ reduction in headache days, or clinically meaningful change in HIT-6, MSQ-RFR, or headache severity at week $24-$ was counted as a responder. HIT-6 6-item Headache Impact Test, MSQ-RFR MigraineSpecific Quality of Life Questionnaire Role FunctionRestrictive

\section{Relationships Among Responder Criteria}

Findings from the linear regression analysis to determine whether the reduction in headache days was driving changes in other outcome measures included a significant association between change from baseline in headache days and change from baseline in HIT- 6 total scores at week 24 (Table 2). Similarly, a significant inverse association was found between change from baseline in headache days and change from baseline in MSQ-RFR scores at week 24 . From both regression analyses, only an approximate $20 \%$ of the variance of the change in the HIT-6 total score and MSQ-RFR domain score could be explained by the observed change in headache days.

Four-way Venn diagrams showing all combinations of responder criteria are presented in Fig. 3, one for onabotulinumtoxinA responders and one for placebo responders. Among onabotulinumtoxinA and placebo responders, a large number of any responders met responder criteria for all four outcomes (onabotulinumtoxinA, $n=140 \quad[20.3 \%] ;$ placebo, $n=60$ [8.6\%]). Patients classified as responders only by
Table 2 Linear regression results at 24 weeks

\begin{tabular}{lrll}
\hline Regression model & Slope & R-squared & $\boldsymbol{P}$ value \\
\hline$\Delta$ HIT-6 score vs. & 0.45 & 0.20 & $<0.0001$ \\
$\Delta$ headache days & & & \\
$\Delta$ MSQ-RFR score vs. & -1.46 & 0.21 & $<0.0001$ \\
$\Delta$ headache days & & & \\
\hline
\end{tabular}

HIT-6 6-item Headache Impact Test, MSQ-RFR Migraine-Specific Quality of Life Questionnaire Role Function-Restrictive

the MSQ-RFR criterion were the most common single-responder group (onabotulinumtoxinA, $n=83 \quad[12.1 \%] ;$ placebo, $n=78 \quad[11.2 \%]$ ). Patients classified as responders by two criteria were common for the combination of MSQ-RFR with HIT- 6 scores and for the combination of $\geq 50 \%$ reduction from baseline in headache days with ADHS scores, but far less common for other two-criteria combinations, each of which paired $a \geq 50 \%$ reduction from baseline in headache days or a severity variable with a quality of life variable. Of the onabotulinumtoxinA responders, $27.3 \%(n=188)$ and $12.9 \%$ $(n=89)$ were identified as headache day nonresponders but met responder criteria on at least one or at least two of the other measures, respectively.

\section{Adverse Events}

AEs for the pooled PREEMPT analysis through week 24 have been published previously [10] and are summarized in Table 3 . Briefly, the frequencies of AEs were similar for both treatment groups, with a greater number reported in patients treated with onabotulinumtoxinA than placebo (Table 3). Most AEs were mild or moderate in severity and resolved without sequelae. Serious AEs were reported for $4.8 \%$ and $2.3 \%$ of patients treated with onabotulinumtoxinA and placebo, respectively. One treatment-related serious $\mathrm{AE}$ was reported in the onabotulinumtoxinA group (hospitalization due to migraine). No new safety or tolerability signals emerged from the pooled study data. 


\section{Onabotulinumtoxin A}

( $n=688 ; \geq 1$ outcome measure, $n=496$;

no outcome measures, $\mathrm{n}=192$ )

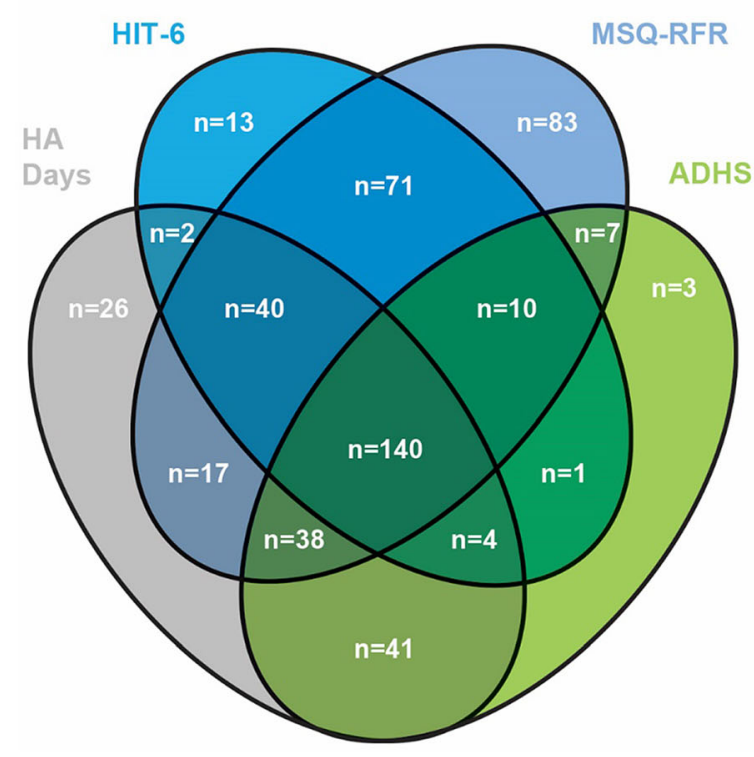

Fig. 3 Number of patients who met all possible responder criteria combinations. Areas defined by the intersections are not proportional. $A D H S$ Average Daily Headache Severity, $H A$ headache, HIT-6 6-item Headache Impact

Table 3 Summary of overall AEs reported in the 24-week, double-blind phase $[10]^{\mathrm{a}}$

\begin{tabular}{lll}
\hline $\boldsymbol{n}(\%)$ & $\begin{array}{l}\text { OnabotulinumtoxinA } \\
(\boldsymbol{n}=\mathbf{6 8 7})\end{array}$ & $\begin{array}{l}\text { Placebo } \\
(\boldsymbol{n}=\mathbf{6 9 2})\end{array}$ \\
\hline All AEs & $429(62.4)$ & $358(51.7)$ \\
TRAEs & $202(29.4)$ & $88(12.7)$ \\
SAEs & $33(4.8)$ & $16(2.3)$ \\
Serious TRAEs & $1(0.1)$ & $0(0.0)$ \\
Discontinuations & $26(3.8)$ & $8(1.2)$ \\
$\quad$ related to AEs & & $0(0.0)$ \\
Death & $0(0.0)$ & \\
\hline
\end{tabular}

$A E$ adverse event, $S A E$ serious adverse event, TRAE treatment-related adverse event

a Reproduced with permission from John Wiley \& Sons Inc. Dodick DW et al., Headache. 2010;50:921-936

\section{Placebo}

( $n=696 ; \geq 1$ outcome measure, $n=394$;

no outcome measures, $n=302$ )

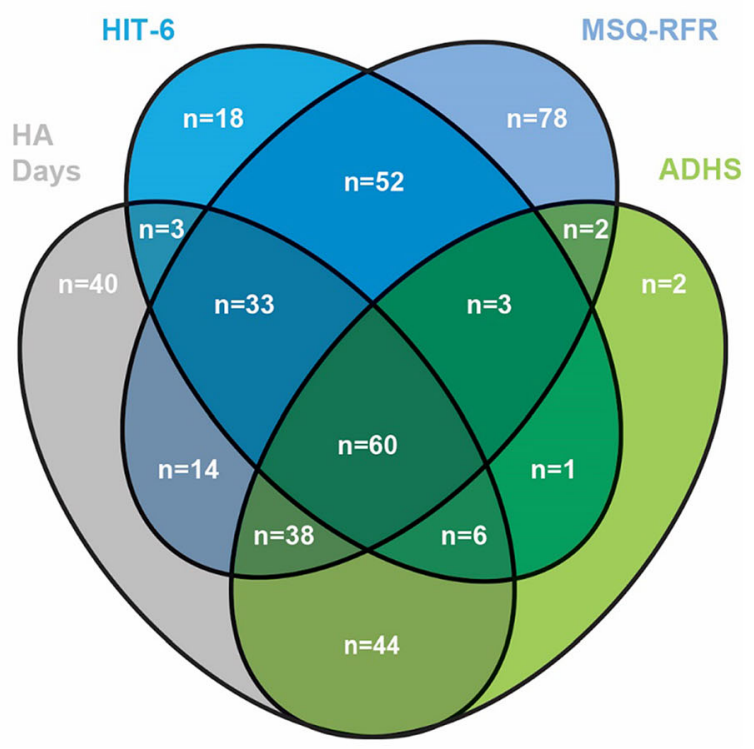

Test, MSQ-RFR Migraine-Specific Quality of Life Questionnaire Role Function-Restrictive

\section{DISCUSSION}

This pooled analysis of data from the PREEMPT clinical trials was designed to determine the extent to which the $50 \%$ responder definition for a reduction in headache day responder status captured the treatment benefits of onabotulinumtoxinA. Due to the phenotype of headache changes in $\mathrm{CM}$ as compared with EM, headache days and not migraine days were the focus of this study. Additional response criteria evaluated in this analysis included clinically meaningful changes in HIT-6 total scores, MSQRFR domain scores, and ADHS scores. OnabotulinumtoxinA treatment provided a significant benefit vs. placebo on improvements on each of these measures, and a greater proportion of patients met responder criteria for at least one outcome measure compared with those who 
had $\mathrm{a} \geq 50 \%$ reduction from baseline in headache days (72.1 vs. $44.8 \%)$. These results demonstrate the importance of using multiple measures to evaluate treatment response in patients with $\mathrm{CM}$.

Significantly higher proportions of onabotulinumtoxinA-treated patients showed improvements vs. placebo-treated patients in terms of headache impact, function, and average daily headache severity, as well as the number of monthly headache days at week 24 . The proportion of onabotulinumtoxinA-treated patients who met at least two of the response criteria was also higher than the proportion of patients who achieved $a \geq 50 \%$ reduction in headache days. Despite the rigor of meeting all four of the criteria, $20.3 \%$ of patients treated with onabotulinumtoxinA did so, compared with $8.6 \%$ of placebo patients; this may be a population of patients who are comprehensive or complete responders.

As this analysis evaluated the simultaneous change in multiple outcome measures, it was important to evaluate the degree to which changes in these outcomes are related to the change in headache days. Hypothetically, the reduction in headache impact could be completely explained by the reduction in monthly headache days alone. However, based on the results from the regression analysis, only $20 \%$ of the variance of the change in the HIT- 6 total score and the MSQ-RFR domain score was explained by the change in headache days, highlighting the complex relationships between headache frequency and other outcomes. A recent, prospective study using the PREEMPT injection protocol found that headache-related disability increased with headache intensity, but not with headache frequency [25]. These findings and the results of our study support the conclusion that these criteria represent distinct measures of treatment response, indicating that there is more to the treatment response than a reduction in headache days.

The Venn diagrams in Fig. 3 provide an overview of the pattern of responder criteria among the PREEMPT population. A large number of onabotulinumtoxinA-treated patients met responder criteria for all four outcome measures, representing a group of comprehensive treatment responders. Interestingly, the next two largest groups were those who met the responder criterion for MSQ-RFR only $(n=83)$ and those who met the responder criteria for MSQ-RFR and HIT-6 $(n=71)$, but not for headache days or ADHS score. This pattern of treatment-related response in the PREEMPT population shows that a non-trivial proportion of patients experience treatment-related benefits that do not overlap with headache day responder status. Specifically, we observed that approximately $27 \%$ of patients treated with onabotulinumtoxinA did not meet headache day responder status but exhibited clinically meaningful benefits on at least one of these outcome measures. Of these, there was a subset of approximately $13 \%$ of patients who met responder criteria on two additional outcomes who require further analysis. Together, these data suggest that it is important for clinicians to review headache days, HIT-6 scores, and MSQRFR data to obtain a fuller picture prior to making a determination about whether a patient is responding to onabotulinumtoxinA or another preventive treatment.

The findings from this study support the conclusion that CM is more complex than just the number of headache days and demonstrate the multidimensional impact of treatment. Therefore, the decision to prescribe a drug to treat CM should not be based only on its ability to provide a reduction in the number of headache days. A patient's decision to continue or terminate treatment is driven by a combination of many clinical outcomes. The European Headache Federation (EHF) guidelines recommend consideration of headache intensity, disability, and patient preferences, in addition to headache day reduction of at least 30\% [26]. Consistent with the EHF guidelines, a quarter of Italian headache physicians surveyed believed that patients achieving $a \geq 30 \%$ reduction in headache days were considered responders (instead of $\geq 50 \%$ reduction), and $40 \%$ of these clinicians believed that more than four treatment cycles were required before classifying a patient as a nonresponder [27]. In agreement with this survey, a real-world observational cohort study using the PREEMPT protocol suggests that approximately $25 \%$ of patients might 
not respond until after four treatment cycles and 1 year, cautioning against adjudicating responder/nonresponder status to avoid missing a significant percentage of patients who might benefit from onabotulinumtoxinA treatment [12]. Real-world data from headache centers in Italy also support the notion that the benefits of onabotulinumtoxinA treatment using the PREEMPT protocol are sustained, even in patients with a recent onset of CM [28]. Preliminary results also suggest that treatment using the PREEMPT protocol may be beneficial to patients with other chronic, difficult-to-treat headache disorders [29].

Understanding the effects of treatment from the patient's perspective is critical to ensuring that the multiple aspects of treatment benefit demonstrated in randomized clinical trials are important and perceptible to patients [30]. As migraine has a significant impact on healthrelated quality of life, disability, mental health, activities of daily living, and work productivity, it is important that guidelines for controlled clinical trials in $\mathrm{CM}$ acknowledge the importance of patient-reported outcome measures to ensure that multidimensional needs of patients are being addressed by treatments [9, 31, 32]. Therefore, we agree with the guidelines of the International Headache Society [9], which state that a reduction in the number of headache days is critical as a primary endpoint in controlled trials, but we also urge clinicians and researchers to collect and select secondary endpoints that reflect multiple aspects of benefit to patients. We also acknowledge that when we broaden the criteria defining therapeutic benefit to become less restrictive, the proportion of individuals meeting those criteria will increase. However, in the current analysis, we believe that we are identifying real responders, as the separation from placebo was maintained in proportions of responders and many participants met at least two criteria. Future studies may also seek to analyze monthly headache days as a continuous variable and consider external references such as satisfaction to further characterize and identify all of the patients who are receiving benefits from treatment.

The limitations of the PREEMPT1 and PREEMPT2 studies, as well as those of the combined pooled analysis, have been discussed $[6,7,10]$; these include the lack of an active comparator (no approved treatment for comparison was available when these studies were conducted) and relatively high placebo response rates. These limitations also apply to the current analysis using patient-reported measures, as well as the possible inherent imprecision of patient-reported outcomes. However, it is critical that treatment benefits are perceptible to patients in order to maintain adherence [30]. In addition, these were exploratory, post hoc analyses without prespecified multiplicity adjustments. However, use of conservative adjustments when considering the significance of treatment differences between treatment arms should adequately control for multiplicity.

\section{CONCLUSIONS}

Measurement of the change in the number of headache days did not fully capture the treatment benefit associated with 24 weeks of onabotulinumtoxinA treatment for CM. When taking into account clinically meaningful improvements of impact, function, and severity, we observed a more accurate reflection of the comprehensive benefit of onabotulinumtoxinA.

\section{ACKNOWLEDGEMENTS}

Funding. Sponsorship for this study and Rapid Service Fee were funded by Allergan (prior to its acquisition by AbbVie).

Authorship. All named authors meet the International Committee of Medical Journal Editors (ICMJE) criteria for authorship for this article, take responsibility for the integrity of the work as a whole, and have given their approval for this version to be published.

Medical Writing, Editorial, and Other Assistance. Writing and editorial assistance was provided to the authors by Sean ShefflerCollins, PhD, at Peloton Advantage, LLC, an 
OPEN Health company, Parsippany, NJ, USA, and was funded by AbbVie. The opinions expressed in this article are those of the authors. The authors received no honorarium/fee or other form of financial support related to the development of this article.

Prior Presentation. Some of the data included in this manuscript were previously presented in poster form at the 19th Congress of the International Headache Society (IHC 2019) September 5-8, 2019, Dublin, Ireland; and the 13th Annual Headache Cooperative of the Pacific (HCOP) Winter Conference January 24-25, 2020, Ojai, CA, USA.

Disclosures. Hans-Christoph Diener, MD, PhD: Received honoraria for participation in clinical trials, contribution to advisory boards or oral presentations in the last 3 years from: Alder, AbbVie, Amgen, Bristol-Myers Squibb, Electrocore, Ipsen Pharma, Eli Lilly, Medtronic, MSD, Novartis, Pfizer, Teva, and Weber \& Weber. Financial support for research projects was provided by AbbVie. HCD serves on the editorial boards of Cephalalgia and Lancet Neurology. HCD co-chairs the Clinical Guidelines Committee of the German Society of Neurology and is member of the Clinical Trials Committee of the International Headache Society. David W. Dodick, MD: Reports the following conflicts within the past 12 months: Consulting: AEON, Amgen, Clexio, Cerecin, AbbVie, Alder, Biohaven, Linpharma, Lundbeck, Promius, Eli Lilly, eNeura, Novartis, Impel, Theranica, WL Gore, Nocira, XoC, Zosano, Upjohn (Division of Pfizer), Pieris, Revance, Equinox. Honoraria: CME Outfitters, Curry Rockefeller Group, DeepBench, Global Access Meetings, KLJ Associates, Majallin LLC, Medlogix Communications, Miller Medical Communications, Southern Headache Society (MAHEC), WebMD Health/Medscape, Wolters Kluwer, Oxford University Press, Cambridge University Press. Research Support: Department of Defense, National Institutes of Health, Henry Jackson Foundation, Sperling Foundation, American Migraine Foundation, Patient Centered Outcomes Research Institute (PCORI). Stock Options/Shareholder/Patents/Board of Directors:
Aural Analytics (options), ExSano (options), Palion (options), Healint (options), Theranica (options), Second Opinion/Mobile Health (options), Epien (options/board), Nocira (options), Ontologics (options/board), King-Devick Technologies (options/board), Precon Health (options/board). Patent 17189376.1-1466:vTitle: Botulinum Toxin Dosage Regimen for Chronic Migraine Prophylaxis. Richard B. Lipton, MD: Serves as consultant or advisory board member or has received honoraria from American Academy of Neurology, Alder, AbbVie, American Headache Society, Amgen, Biohaven, Biovision, Boston Scientific, Dr. Reddy's, Electrocore, Eli Lilly, eNeura Therapeutics, GlaxoSmithKline, Merck, Pernix, Pfizer, Teva, Trigemina, Vector, and Vedanta. Holds stock options in eNeura and Biohaven. Aubrey Manack Adams, PhD and Ronald E. DeGryse, MS, MA: Full-time employees of AbbVie and may hold stock in AbbVie. Stephen D. Silberstein, MD: Consultant and/or advisory panel member for and has received honoraria from Alder Biopharmaceuticals, AbbVie, Amgen, Avanir, eNeura, ElectroCore Medical, Labrys Biologics, Medscape, Medtronic, Neuralieve, NINDS, Pfizer, and Teva. His employer receives research support from AbbVie, Amgen, Cumberland Pharmaceuticals, ElectroCore Medical, Labrys Biologics, Eli Lilly, Mars, and Troy Healthcare.

Compliance with Ethics Guidelines. Both trials discussed in this article were conducted in accordance with the Declaration of Helsinki Code of Federal Regulations and Good Clinical Practices and US requirements of public registration (NCT00156910 [PREEMPT 1] and NCT00168428 [PREEMPT 2]). Investigators obtained approval from the independent ethics committee or local institutional review board at each investigational site prior to study initiation. Written informed consent was obtained from each randomized patient.

Data Availability. Data reported in this manuscript are available within the article and/ or its supplementary materials. AbbVie will share de-identified patient-level data and/or study-level data, including protocols and clinical study reports, for phase $2-4$ trials completed 
after 2008 that are registered on ClinicalTrials .gov or EudraCT. The indication studied in the trial must have regulatory approval in the United States and/or the European Union and the primary manuscript from the trial must be published prior to data sharing. To request access to the data, the researcher must sign a data use agreement. All shared data are to be used for non-commercial purposes only. More information can be found on https://www. allerganclinicaltrials.com/PatientDataRequest. htm.

Open Access. This article is licensed under a Creative Commons Attribution-NonCommercial 4.0 International License, which permits any non-commercial use, sharing, adaptation, distribution and reproduction in any medium or format, as long as you give appropriate credit to the original author(s) and the source, provide a link to the Creative Commons licence, and indicate if changes were made. The images or other third party material in this article are included in the article's Creative Commons licence, unless indicated otherwise in a credit line to the material. If material is not included in the article's Creative Commons licence and your intended use is not permitted by statutory regulation or exceeds the permitted use, you will need to obtain permission directly from the copyright holder. To view a copy of this licence, visit http://creativecommons.org/licenses/by$\mathrm{nc} / 4.0 /$.

\section{REFERENCES}

1. Blumenfeld AM, Varon SF, Wilcox TK, et al. Disability, HRQoL and resource use among chronic and episodic migraineurs: results from the International Burden of Migraine Study (IBMS). Cephalalgia. 2011;31:301-15.

2. Lanteri-Minet M, Duru G, Mudge M, Cottrell S. Quality of life impairment, disability and economic burden associated with chronic daily headache, focusing on chronic migraine with or without medication overuse: a systematic review. Cephalalgia. 2011;31:837-50.

3. Headache Classification Committee of the International Headache Society. The International
Classification of Headache Disorders, 3rd edition. Cephalalgia. 2018;38:1-211.

4. Lipton RB, Manack Adams A, Buse DC, Fanning KM, Reed ML. A comparison of the Chronic Migraine Epidemiology and Outcomes (CaMEO) Study and American Migraine Prevalence and Prevention (AMPP) Study: demographics and headache-related disability. Headache. 2016;56: 1280-9.

5. Buse DC, Manack A, Serrano D, Turkel C, Lipton RB. Sociodemographic and comorbidity profiles of chronic migraine and episodic migraine sufferers. J Neurol Neurosurg Psychiatry. 2010;81:428-32.

6. Aurora SK, Dodick DW, Turkel CC, et al. OnabotulinumtoxinA for treatment of chronic migraine: results from the double-blind, randomized, placebo-controlled phase of the PREEMPT 1 trial. Cephalalgia. 2010;30:793-803.

7. Diener HC, Dodick DW, Aurora SK, et al. OnabotulinumtoxinA for treatment of chronic migraine: results from the double-blind, randomized, placebo-controlled phase of the PREEMPT 2 trial. Cephalalgia. 2010;30:804-14.

8. Tfelt-Hansen P, Block G, Dahlof C, et al. Guidelines for controlled trials of drugs in migraine: second edition. Cephalalgia. 2000;20:765-86.

9. Tassorelli C, Diener HC, Dodick DW, et al. Guidelines of the International Headache Society for controlled trials of preventive treatment of chronic migraine in adults. Cephalalgia. 2018;38:815-32.

10. Dodick DW, Turkel CC, DeGryse RE, et al. OnabotulinumtoxinA for treatment of chronic migraine: pooled results from the double-blind, randomized, placebo-controlled phases of the PREEMPT clinical program. Headache. 2010;50:921-36.

11. Matharu M, Halker R, Pozo-Rosich P, DeGryse R, Manack Adams A, Aurora SK. The impact of onabotulinumtoxinA on severe headache days: PREEMPT 56-week pooled analysis. J Headache Pain. 2017;18:78.

12. Vernieri F, Paolucci M, Altamura C, et al. Onabotulinumtoxin-A in chronic migraine: should timing and definition of non-responder status be revised? Suggestions from a real-life Italian multicenter experience. Headache. 2019;59:1300-9.

13. American Headache Society. The American Headache Society position statement on integrating new migraine treatments into clinical practice. Headache. 2019;59:1-18.

14. Headache Classification Subcommittee of the International Headache Society. The international 
classification of headache disorders, 2nd ed. Cephalalgia. 2004;24(Suppl 1):9-160.

15. Kosinski M, Bayliss MS, Bjorner JB, et al. A six-item short-form survey for measuring headache impact: the HIT-6. Qual Life Res. 2003;12:963-74.

16. Jhingran P, Davis SM, LaVange LM, Miller DW, Helms RW. MSQ: Migraine-Specific Quality-of-Life Questionnaire. Further investigation of the factor structure. Pharmacoeconomics. 1998;13:707-17.

17. Rendas-Baum R, Bloudek LM, Maglinte GA, Varon SF. The psychometric properties of the MigraineSpecific Quality of Life Questionnaire version 2.1 (MSQ) in chronic migraine patients. Qual Life Res. 2013;22:1123-33.

18. Silberstein SD, Dodick DW, Aurora SK, et al. Per cent of patients with chronic migraine who responded per onabotulinumtoxinA treatment cycle: PREEMPT. J Neurol Neurosurg Psychiatry. 2015;86:996-1001.

19. Coeytaux RR, Kaufman JS, Chao R, Mann JD, Devellis RF. Four methods of estimating the minimal important difference score were compared to establish a clinically significant change in Headache Impact Test. J Clin Epidemiol. 2006;59: 374-80.

20. Rendas-Baum R, Yang M, Varon SF, Bloudek LM, DeGryse RE, Kosinski M. Validation of the Headache Impact Test (HIT-6) in patients with chronic migraine. Health Qual Life Outcomes. 2014;12:117.

21. Dodick DW, Silberstein S, Saper J, et al. The impact of topiramate on health-related quality of life indicators in chronic migraine. Headache. 2007;47: 1398-408.

22. Dodick DW, Turkel CC, DeGryse RE, et al. Assessing clinically meaningful treatment effects in controlled trials: chronic migraine as an example. J Pain. 2015;16:164-75.

23. Speck RM, Shalhoub H, Wyrwich KW, et al. Psychometric validation of the Role Function Restrictive domain of the Migraine Specific Quality-of-Life Questionnaire version 2.1 electronic patient- reported outcome in patients with episodic and chronic migraine. Headache. 2019;59:756-74.

24. Aurora SK, Winner P, Freeman MC, et al. OnabotulinumtoxinA for treatment of chronic migraine: pooled analyses of the 56-week PREEMPT clinical program. Headache. 2011;51:1358-73.

25. Torres-Ferrus M, Gallardo VJ, Alpuente A, PozoRosich P. Influence of headache pain intensity and frequency on migraine-related disability in chronic migraine patients treated with onabotulinumtoxinA. J Headache Pain. 2020;21:88.

26. Bendtsen L, Sacco S, Ashina M, et al. Guideline on the use of onabotulinumtoxinA in chronic migraine: a consensus statement from the European Headache Federation. J Headache Pain. 2018;19:91.

27. Tassorelli $C$, Aguggia $M$, De Tommaso $M$, et al. Onabotulinumtoxin A for the management of chronic migraine in current clinical practice: results of a survey of sixty-three Italian headache centers. J Headache Pain. 2017;18:66.

28. Ornello R, Guerzoni S, Baraldi C, et al. Sustained response to onabotulinumtoxin $\mathrm{A}$ in patients with chronic migraine: real-life data. J Headache Pain. 2020;21:40.

29. Lampl C, Rudolph M, Bräutigam E. OnabotulinumtoxinA in the treatment of refractory chronic cluster headache. J Headache Pain. 2018;19:45.

30. Haywood KL, Mars TS, Potter R, Patel S, Matharu M, Underwood M. Assessing the impact of headaches and the outcomes of treatment: a systematic review of patient-reported outcome measures (PROMs). Cephalalgia. 2018;38:1374-86.

31. Tfelt-Hansen $\mathrm{P}$, Pascual J, Ramadan $\mathrm{N}$, et al. Guidelines for controlled trials of drugs in migraine: third edition. A guide for investigators. Cephalalgia. 2012;32:6-38.

32. Silberstein S, Tfelt-Hansen P, Dodick DW, et al. Guidelines for controlled trials of prophylactic treatment of chronic migraine in adults. Cephalalgia. 2008;28:484-95. 